\title{
The Equivalent of the Goldbach Conjecture and "Even Number Is the Difference Between Two Prime Numbers"
}

\author{
Mi Zhou ${ }^{1,2}$ \\ ${ }^{1}$ Philosophy Department, Ren Min University of China, Beijing, China \\ ${ }^{2}$ Commerce Department, Suqian Economic and Trade Vocational School, Suqian, China
}

\section{Email address:}

Zhoumi19920626@163.com

\section{To cite this article:}

Mi Zhou. The Equivalent of the Goldbach Conjecture and "Even Number Is the Difference Between Two Prime Numbers". Science Discovery. Vol. 7, No. 3, 2019, pp. 161-164. doi: 10.11648/j.sd.20190703.15

Received: April 9, 2019; Accepted: May 25, 2019; Published: June 15, 2019

\begin{abstract}
Goldbach conjecture can be described as "an even number is the sum of two prime Numbers,", this description is well known, the Canadian guy's book «the unresolved problems in number theory», put forward a conjecture which is contrary to goldbach: that "evens are the different between two prime Numbers", the difficulty of the conjecture is not less than goldbach conjecture, as also an unresolved problem.This paper, based on the promotion of chandra symmetric matrix, there is a natural number as long as there is any matrix at the same time don't appear in the matrix as well as the matrix beginning of 4 , thus making the conjecture set up, thus obtained the equivalent propositions of "an even number are the difference between the two primes" conjecture: $2 m n+m+n$ and $2 m+m+n+x(m, n$ for any natural number, $x$ takes only one value at a time, is a fixed) Is these two formulas can show all the natural Numbers greater than $4+x$ ? If not, then the "even number is the difference between two prime Numbers" conjecture is true, which is the equivalent of the conjecture. And I get the equivalent proposition of golabach too! Mathematicians can turn to this description, as long as have the solution of this new description, the original conjecture will also be solved, the road of research also greatly broaden, mathematicians on the new description of the solution, in the process of research should be have some achievements. The difference between this equivalent proposition and the original conjecture is that the original conjecture is only a description of a concept, while the equivalent proposition tends to be digitized and formulated.
\end{abstract}

Keywords: Number Theory, Equivalent Proposition, Chandra Matrix

\section{“偶数为两个质数的差”猜想的等价命题}

\author{
周密 1,2 \\ ${ }^{1}$ 中国人民大学哲学院, 北京, 中国 \\ 2宿迁经贸高等职业技术学校商贸系, 宿迁, 中国
}

邮箱

Zhoumi19920626@163.com

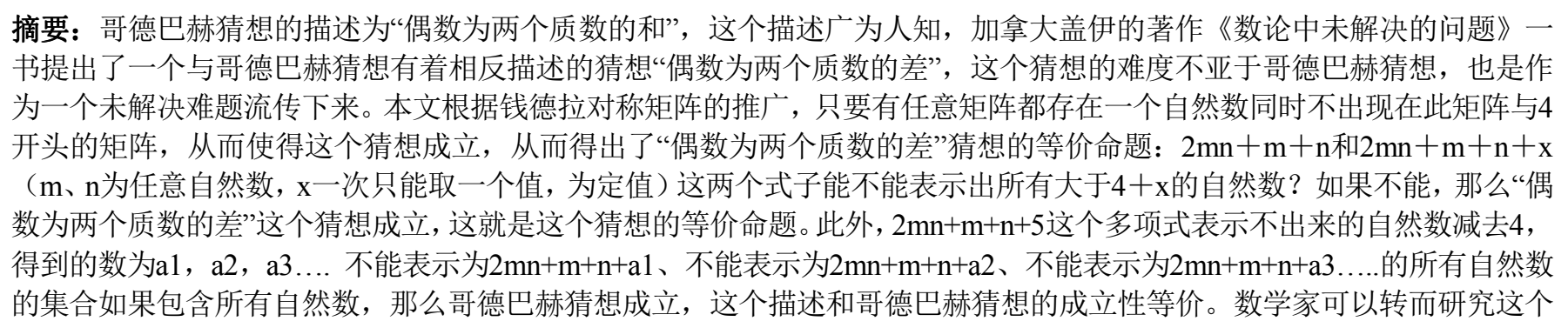


描述, 只要解决了这个新的描述, 这个原来猜想也就解决, 研究之路也极大拓宽, 不排除有数学家在新的描述上解决的可 能性, 就算解决不了, 在研究的过程中也当有所发现。这个等价命题与原猜想的区别在于, 原来的猜想只是一个概念的描 述, 等价命题则倾向于数字化, 公式化。除此以外, 本文也得到了哥德巴赫猜想的等价命题。

关键词: 数论, 等价命题, 钱德拉矩阵

\section{1. 引言}

哥德巴赫猜想广为人知, 它的描述就是“偶数为两个 质数的和”, 也就是陈景润的“1 1 1”版本。然而一开始哥 德巴赫提出这个猜想的时候, 它的描述并不是这个, 它 的最初描述就是 “大于 9 的奇数为三个质数的和”, 是欧 拉把哥德巴赫提出的这个描述也就是 “奇哥猜”转化为现 在流行的版本 “偶哥猜”, 欧拉的等价版本提出来之后, 像陈景润, 王元, 潘承洞这些人的成果都基于欧拉的新 版本, 欧拉的工作意义就在于此, 极大拓宽了研究视野。 加拿大盖伊所著《数论中未解决的问题》 [1]一书收录了 数论里数百个未解决问题, 其中有一个猜想与哥德巴赫 猜想 [2]的描述相反, 它的描述为“偶数为两个质数的 差”[3]。我把这个猜想通过钱德拉矩阵的推广, 也把这个 猜想做了一个转化, 转换为另外一个描述, 除此以外本 文也得到了哥德巴赫猜想的等价命题, 这两个猜想的研 究者穷尽了已有的办法, 收效不大, 我找到了这个猜想 的等价命题, 把它转化成了另外一个描述, 数学家可以 转而研究这个描述, 只要解决了这个新的描述, 这个原 来猜想也就解决, 研究之路也极大拓宽, 不排除有数学 家在新的描述上解决的可能性, 就算解决不了, 在研究 的过程中也当有所发现。

这个等价命题与原猜想的区别在于, 原来的猜想只是 一个概念的描述, 等价命题则倾向于数字化, 公式化。

\section{2. 方法}

钱德拉矩阵有一个特殊的性质, 就是一个自然数 $\mathrm{N}$ 出 现在里面, $2 \mathrm{~N}+1$ 必为合数, 反之则为质数, 本文独创钱 德拉矩阵的推广, 得出了无数具有相似性质的矩阵, 只要
有任意一个推广的矩阵和原来的矩阵同时有至少一个相 同的自然数不出现在这两个矩阵里, “偶数为两个质数的 差”猜想成立。但是这个猜想的成立性必须同时满足两个 条件：1.两个矩阵存在至少一个相同的自然数; 2 . 这个自 然数同时不出现。根据这两个条件限制, 从而衍生出了“偶 数为两个质数的差" 猜想的等价命题。除此以外, 也可以 得到哥德巴赫猜想的等价命题。

\section{1. 一个未解决难题}

哥德巴赫猜想的描述为“偶数为两个质数的和”, 加拿 大学者盖伊出版的著作《数论中未解决的问题》记载了一 个与哥德巴赫猜想相反的猜想: 偶数为两个质数的差。

\section{2. 钱德拉对称矩阵}

1934年，东印度（现孟加拉国）的钱德拉提出一个正 方形篮子:

第 1 行是首项为 4 , 公差为 3 的等差数列 $4,7,10, \ldots$, $4+3(n-1), \ldots$

第 2 行是首项为 7 , 公差为 5 的等差数列 $7,12,17, \ldots$, $7+5(\mathrm{n}-1), \ldots$

第 3 行是首项为 10 , 公差为 7 的等差数列 $10,17,24, \ldots$, $10+7(\mathrm{n}-1), \ldots$

第 4 行是首项为 13 , 公差为 9 的等差数列 $13,22,31, \ldots$, $13+9(\mathrm{n}-1), \ldots$

第 $m$ 行是首项为 $3 m+1$, 公差为 $2 m+1$ 的等差数列 $3 m+1,5 m+2,7 m+3, \ldots$, 它 的 第 $n$ 项 为 $3 m+1+(n-1)(2 m+1)=2 m n+m+n, \ldots$

写成以下的阵列:

表1 钱德拉矩阵。

\begin{tabular}{|c|c|c|c|c|c|c|c|c|}
\hline & 第1列 & 第2列 & 第3列 & 第4列 & 第5列 & $\ldots$ & 第n列 & $\ldots$ \\
\hline 第1行 & 4 & 7 & 10 & 13 & 16 & $\ldots$ & $4+3(n-1)$ & $\ldots$ \\
\hline 第2行 & 7 & 12 & 17 & 22 & 27 & $\ldots$ & $7+5(n-1)$ & $\ldots$ \\
\hline 第3行 & 10 & 17 & 24 & 31 & 38 & $\ldots$ & $10+7(n-1)$ & $\ldots$ \\
\hline 第4行 & 13 & 22 & 31 & 40 & 49 & $\ldots$ & $13+9(n-1)$ & $\ldots$ \\
\hline 第5行 & 16 & 27 & 38 & 49 & 60 & $\ldots$ & $16+11(n-1)$ & $\ldots$ \\
\hline$\ldots$ & $\ldots$ & $\ldots$ & $\ldots$ & $\ldots$ & $\ldots$ & $\ldots$ & $\ldots$ & $\ldots$ \\
\hline 第m行 & $3 m+1$ & $5 m+2$ & $7 m+3$ & $9 m+4$ & $11 \mathrm{~m}+5$ & $\ldots$ & $2 m n+m+n$ & $\ldots$ \\
\hline$\ldots$ & $\ldots$ & $\ldots$ & $\ldots$ & $\ldots$ & $\ldots$ & $\ldots$ & $\ldots$ & $\ldots$ \\
\hline
\end{tabular}

\section{3. 钱德拉篮子的性质[5]证明 $[6]$}

这个方篮的奥妙在于: 如果某个自然数 $\mathrm{N}$ 出现在表中, 那么 $2 \mathrm{~N}+1$ 肯定不是素数; 如果 $\mathrm{N}$ 在表中不出现, 那么 $2 \mathrm{~N}$ +1 肯定是素数。
事 实上, 如 果 $\mathrm{N}=2 \mathrm{mn}+\mathrm{m}+\mathrm{n}$, 则 $2 \mathrm{~N}+1=2(2 \mathrm{mn}+\mathrm{m}+\mathrm{n})+1=4 \mathrm{mn}+2 \mathrm{~m}+2 \mathrm{n}+1=(2 \mathrm{~m}+1)(2 \mathrm{n}+1)$, 它 不是素数。反之, 设 $\mathrm{N}$ 在表中不出现, 如果 $2 \mathrm{~N}+1$ 不是素数, 则 $2 \mathrm{~N}+1$ 必定是两个奇数之积, 写 $2 \mathrm{~N}+1=(2 \mathrm{~m}+1)(2 \mathrm{n}+1)=4 \mathrm{mn}+2 \mathrm{~m}+2 \mathrm{n}+1$, 得到 $\mathrm{N}=2 \mathrm{mn}+\mathrm{m}+\mathrm{n}$, 
它出现在表中, 与假设矛盾。所以当 $\mathrm{N}$ 不出现在矩阵中时 $2 \mathrm{~N}+1$ 必为质数。

\section{4. 钱德拉篮子的推广 [7]}

据此做出了几个类似矩阵 (简化一些) , 对它做出了 推演, 找到了无数个具有相同性质的矩阵。这个矩阵推演 后，会有很多美妙的现象出现以及有有效的应用。

表2 5 开头的矩阵。

\begin{tabular}{lllllll}
\hline 5 & 8 & 11 & 14 & 17 & 20 & $\ldots \ldots \ldots \ldots$ \\
8 & 13 & 18 & 23 & 28 & 33 & $\ldots \ldots \ldots \ldots$ \\
11 & 18 & 25 & 32 & 39 & 46 & $\ldots \ldots \ldots \ldots$ \\
$\ldots \ldots \ldots$ & & & & & & \\
\hline
\end{tabular}

在此矩阵中 [8]若干自然数 $\mathrm{N}$ 出现在此矩阵中则 $2 \mathrm{~N}-1$ 肯定不是质数, 若不出现则 $2 \mathrm{~N}-1$ 必然为质数, 因为第一 个矩阵 5 不出现, 第二个矩阵 6 不出现而 $2 \times 5+1=2 \times 6-1$, 它们表示的是相同的自然数所以成立。也就是说, 出现在 这个矩阵里的自然数都可以表示为 $2 \mathrm{mn}+\mathrm{m}+\mathrm{n}+1$ 设 $\mathrm{N}$ 在表 中不出现, 如果 $2 \mathrm{~N}-1$ 不是素数, 则 $2 \mathrm{~N}-1$ 必定是两个奇 数之积, 写成 $2 \mathrm{~N}-1=(2 \mathrm{~m}+1)(2 \mathrm{n}+1)=4 \mathrm{mn}+2 \mathrm{~m}+2 \mathrm{n}+2$, 得到 $\mathrm{N}=2 \mathrm{mn}+\mathrm{m}+\mathrm{n}+1$, 它出现在表中, 与假设矛盾。所以当 $\mathrm{N}$ 不出现在矩阵中时 $2 \mathrm{~N}-1$ 必为质数。

同理, 再列出一个矩阵:

表3 6开头的矩阵。

\begin{tabular}{llllll}
\hline 6 & 9 & 12 & 15 & 18 & $\ldots \ldots \ldots \ldots$ \\
9 & 14 & 19 & 24 & 29 & $\ldots \ldots \ldots \ldots$ \\
12 & 19 & 26 & 33 & 40 & $\ldots \ldots \ldots$. \\
$\ldots \ldots \ldots$ & & & & & \\
\hline
\end{tabular}

可得出若自然数 $\mathrm{N}$ 出现在此矩阵中则 $2 \mathrm{~N}-3$ 肯定不是 质数, 若不出现则 $2 \mathrm{~N}-3$ 必为质数, 因为出现在这个矩阵 里的自然数都可以表示为 $2 \mathrm{mn}+\mathrm{m}+\mathrm{n}+2$ 设 $\mathrm{N}$ 在表中不出现, 如果 $2 \mathrm{~N}-3$ 不是素数, 则 $2 \mathrm{~N}-3$ 必定是两个奇数之积, 写 成 $2 \mathrm{~N}-3=(2 \mathrm{~m}+1)(2 \mathrm{n}+1)=4 \mathrm{mn}+2 \mathrm{~m}+2 \mathrm{n}+3$, 得 到 $\mathrm{N}=2 \mathrm{mn}+\mathrm{m}+\mathrm{n}+2$, 它出现在表中, 与假设矛盾。所以当 $\mathrm{N}$ 不出现在矩阵中时 $2 \mathrm{~N}-3$ 必为质数。

还可列出:

表4 4+x开头的矩阵。

\begin{tabular}{lllll}
\hline $4+\mathrm{x}$ & $7+\mathrm{x}$ & $10+\mathrm{x}$ & $13+\mathrm{x}$ & $\ldots \ldots \ldots$ \\
$7+\mathrm{x}$ & $12+\mathrm{x}$ & $17+\mathrm{x}$ & $22+\mathrm{x}$ & $\ldots \ldots \ldots \ldots$ \\
$10+\mathrm{x}$ & $17+\mathrm{x}$ & $24+\mathrm{x}$ & $31+\mathrm{x}$ & $\ldots \ldots \ldots \ldots$ \\
$\ldots \ldots \ldots$ & & & \\
\hline
\end{tabular}

可得出若自然数 $\mathrm{N}$ 出现在矩阵中则 $2 \mathrm{~N}-(2 \mathrm{x}-1)$ 肯 定不是质数, 若不出现则 $2 \mathrm{~N}-(2 \mathrm{x}-1)$ 肯定是质数。因 为出现在这个矩阵里的自然数都可以表示为 $2 m n+m+n+x$ 设 $\mathrm{N}$ 在表中不出现, 如果 $2 \mathrm{~N}-(2 \mathrm{x}-1)$ 不是素数, 则 $2 \mathrm{~N}$ - $(2 \mathrm{x}-1)$ 必定是两个奇数之积, 写成 $2 \mathrm{~N}-(2 \mathrm{x}-1)$ $=(2 m+1)(2 n+1)=4 m n+2 m+2 n+x$, 得到 $N=2 m n+m+n+x$, 它 出现在表中, 与假设矛盾。所以当 $\mathrm{N}$ 不出现在矩阵中时 $2 \mathrm{~N}$ $-(2 x-1)$ 必为质数。

\section{5. 得出猜想的等价命题[9]}

以 $4 、 5 、 6 、 7 、 8 、 9 、 10 、 11 \ldots$ 开头的矩阵的矩对应 的分别是 $2 n+1 、 2 n-1 、 2 n-3 、 2 n-5 、 2 n-7 、 2 n-9 、 2 n-11$ 、 $2 n-13 \ldots . .$. 列一个表[9]:

表5 对应表。

\begin{tabular}{lll}
\hline 4 & $2 \mathrm{n}+1$ & \\
5 & $2 \mathrm{n}-1$ & $(2 \mathrm{n}+1)-2$ \\
6 & $2 \mathrm{n}-3$ & $(2 \mathrm{n}+1)-4$ \\
7 & $2 \mathrm{n}-5$ & $(2 \mathrm{n}+1)-6$ \\
8 & $2 \mathrm{n}-7$ & $(2 \mathrm{n}-+1)-8$ \\
9 & $2 \mathrm{n}-9$ & $(2 \mathrm{n}+1)-10$ \\
10 & $2 \mathrm{n}-11$ & $(2 \mathrm{n}+1)-12$ \\
11 & $2 \mathrm{n}-13$ & $(2 \mathrm{n}+1)-14$ \\
$\ldots \ldots \ldots$ & & \\
\hline
\end{tabular}

当 $\mathrm{n}$ 不出现在 4 开头的矩阵里的时候, $2 n+1$ 是质数, 那么当 $\mathrm{n}$ 同时不出现在 4 和 5 开头的矩阵的时候 $2 n+1$ 和 $2 n$ -1 为质数, 这个时候 $2=(2 n+1)-(2 n-1)=$ 质数一质 数。

当 $\mathrm{n}$ 不出现在 4 开头的矩阵里的时候, $2 \mathrm{n}+1$ 是质数, 那么当 $\mathrm{n}$ 同时不出现在 4 和 6 开头的矩阵的时候 $2 n+1$ 和 $2 n-3$ 为质数, 所以 $4=(2 n+1)-(2 n-3)=$ 质数-质数。

当 $\mathrm{n}$ 不出现在 4 开头的矩阵里的时候, $2 \mathrm{n}+1$ 是质数, 那么当 $\mathrm{n}$ 同时不出现在 4 和 7 开头的矩阵的时候 $2 n+1$ 和 $2 n-5$ 是质数, 所以 $6=(2 n+1)-(2 n-5)=$ 质数-质数。

以此类推, 对于同时不出现在 4 和 $4+x$ 开头的矩阵里的 $\mathrm{n}, 2 \mathrm{n}+1$ 和 $2 \mathrm{n}-(2 \mathrm{x}-1)$ 都是质数, 这个时候 $2 \mathrm{x}$ 等 $=(2 \mathrm{n}$ $+1)-(2 n-2 x+1)$, 显然 $2 x$ 可以表示出所有偶数。

\section{3. 结果}

这个猜想前提是至少存在一个自然数 $n$ 同时不出现在 4 开头和 $4+x$ 开头的矩阵, 4 和 $4+x$ 开头的矩阵都有无数个 自然数不出现, 只要有一个是相同的, 这个猜想就成立了, 这个猜想很大可能是成立的。[10]出现在4开头的自然数, 都可以被 $2 m n+m+n$ 这个式子表示出来, 出现在 $4+x$ 矩阵 的自然数, 都可以被 $2 m n+m+n+x$ 这个式子表示出来, 也就是说有一个大于 $4+\mathrm{x}$ 自然数同时不能能被 $2 \mathrm{mn}+\mathrm{m}+$ $n$ 和 $2 m n+m+n+x$ 这两个式子表示出来, 那么这个自然数 就不出现在 4 和 $4+\mathrm{x}$ 开头的这两个矩阵里, 偶数为两个质 数的差这个猜想就成立。

\section{1. 得出等价命题方案的提炼}

上面关于等价命题的提出是基于钱德拉对称矩阵，它 的优点是比较直观, 缺点是比较冗长。下面还有一个比较 简洁明了的等价命题得出方案, 是从钱德拉对称矩阵里提 炼出来的。

\section{2. 提炼版等价命题的提出方案}

当 $\mathrm{N}=2 \mathrm{mn}+\mathrm{m}+\mathrm{n}$ 的时候, $2 \mathrm{~N}+1=2(2 \mathrm{mn}+\mathrm{m}+\mathrm{n})+1=(2 \mathrm{~m}+1)$ $(2 n+1)$, 是合数。当 $\mathrm{N}$ 不等于 $2 \mathrm{mn}+\mathrm{m}+\mathrm{n}$ 的时候, $2 \mathrm{~N}+1$ 是质数。 
假设 $\mathrm{N} 1$ 可以表示为 $2 \mathrm{mn}+\mathrm{m}+\mathrm{n}, \mathrm{N} 2$ 可以表示为 $2 \mathrm{mn}+\mathrm{m}+\mathrm{n}+\mathrm{x}$, 那么 $2 \mathrm{~N} 1+1=2(2 \mathrm{mn}+\mathrm{m}+\mathrm{n})+1=(2 \mathrm{~m}+1)(2 \mathrm{n}+1)$, 是合数; $2 \mathrm{~N} 2-(2 \mathrm{x}-1)=2(2 \mathrm{mn}+\mathrm{m}+\mathrm{n}+\mathrm{x})-(2 \mathrm{x}-1)=$ $(2 \mathrm{~m}+1)(2 \mathrm{n}+1)$, 是合数。当 $\mathrm{N} 1$ 不可以表示为 $2 \mathrm{mn}+\mathrm{m}+\mathrm{n}$ 的时候, $2 \mathrm{~N} 1+1$ 是质数, 当 $\mathrm{N} 2$ 不可以表示为 $2 \mathrm{mn}+\mathrm{m}+\mathrm{n}+\mathrm{x}$ 的时候, $2 \mathrm{~N} 2-(2 \mathrm{x}-1)$ 是质数。当 $\mathrm{N} 1=\mathrm{N} 2$, 且同时不 可以表示为 $2 m n+m+n$ 和 $2 m n+m+n+x$ 的时候, $2 \mathrm{~N} 1+1$ 和 $2 \mathrm{~N} 2$ $-(2 \mathrm{x}-1)$ 都是质数, 且 $(2 \mathrm{~N} 1+1)-(2 \mathrm{~N} 2-2 \mathrm{x}+1)=2 \mathrm{x}$, $2 x$ 是偶数, 为两个质数的差。

\section{3. 哥德巴赫猜想的等价命题}

对于不出现在 $4+x$ 开头矩阵的 $\mathrm{N}, 2 \mathrm{~N}-(2 \mathrm{X}-1)=\mathrm{P}, \mathrm{P}$ 是质 数, 只要 $2 \mathrm{x}-1$ 也是质数, $2 \mathrm{~N}$ 就是两个质数的和, 对于哥猜 成立有两个条件, $1,2 \mathrm{x}-1$ 为质数, $2,2 \mathrm{~N}$ 能够表示出所有 偶数。对于条件 1 的成立, 只要让 $\mathrm{x}$ 不出现在 5 开头的矩阵 里就行, 此时 $2 \mathrm{x}-1$ 为质数, 也就是说 $\mathrm{x}$ 不能表示为 $2 m n+m+n+1$, 此时 $N$ 不出现在 $4+x$ 里面, 也就是说 $N$ 不出 现在: 不出现在 5 开头矩阵里的自然数加上 4 得到的自然数 也就是不出现在 9 开头的矩阵里的自然数集合为 $\mathrm{A}$, 以 $\mathrm{A}$ 里 的自然数开头的矩阵, 不出现在这些矩阵力的自然数为 $\mathrm{B}$, 只要 $\mathrm{B}$ 能够表示出所有自然数, 哥德巴赫猜想就成立。

结论: $2 m n+m+n+5$ 这个多项式表示不出来的自然数 减去 4 , 得到的数为 $a 1, a 2, a 3 \ldots$ 不能表示为 $2 m n+m+n+a 1$ 、 不能表示为 $2 m n+m+n+a 2$ 、不能表示为 $2 m n+m+n+a 3 \ldots$. 的所有自然数的集合如果包含所有自然数, 那么哥德巴赫 猜想成立, 这个描述和哥德巴赫猜想的成立性等价。

\section{4. 讨论}

利用钱德拉对称矩阵得出 “偶数为两个质数的差” 猜 想的等价命题, 优点是比较直观, 缺点是比较冗长; 提炼 版本的等价命题提出方案, 优点是比较简洁, 缺点是比较 䀲涩。大家觉得哪种方案更好呢?

\section{5. 结论}

1.“偶数为两个质数的差”猜想的等价命题: $2 \mathrm{mn}+\mathrm{m}$ $+n$ 和 $2 m n+m+n+x(m 、 n$ 为任意自然数, $x$ 一次只能取 一个值, 为定值) 这两个式子能不能表示出所有大于 $4+x$
的自然数? 如果不能, 那么 “偶数为两个质数的差”这个猜 想成立, 这就是这个猜想的等价命题。

2、 $2 m n+m+n+5$ 这个多项式表示不出来的自然数减 去 4 , 得到的数为 $\mathrm{a} 1, \mathrm{a} 2, \mathrm{a} 3 \ldots$ 不能表示为 $2 m n+m+n+a 1$ 、 不能表示为 $2 m n+m+n+a 2$ 、不能表示为 $2 m n+m+n+a 3 \ldots$. 的所有自然数的集合如果包含所有自然数, 那么哥德巴 赫猜想成立, 这个描述和哥德巴赫猜想的成立性等价。

\section{致谢}

本文感谢加拿大渥太华大学黄骏教授以及宿迁学院 相关老师的研讨论证和帮助。

\section{参考文献}

[1] 盖伊. 《数论中未解决的问题》. 2007 年 1 月 4 日, 科学出 版社。

[2]＼cjkstart哥德巴赫猜想.1742

年.https://baike.so.com/doc/5351515-5586973.html。

[3] 偶数为两个质数的差. 《数论中未解决的问题》. 2007 年 1 月 4 日, 科学出版社。

[4] 钱德拉对称矩阵.百度百

科.https://baike.so.com/doc/24705238-25608647.html。

[5] 钱德拉笁子的性质.百度百

科.https://baike.so.com/doc/24705238-25608647.html。

[6] 钱德拉篮子的性质证明.周密. 《钱德拉篮及其推广》.中国 科学院《科学智慧火花》

http://idea.cas.cn/viewdoc.action?docid=20993。

[7] 钱德拉笚子的推广.周密.中国科学院《科学智慧火花》 $\mathrm{http}: / /$ idea.cas.cn/viewdoc.action?docid=20993。

[8] 推广矩阵的特性.周密.中国科学院《科学智慧火花》 http://idea.cas.cn/viewdoc.action?docid=20993。

[9] 等价命题.百度百

科.https://baike.so.com/doc/8585945-8906762.html。

[10] “偶数为两个质数的差”很大可能成立, 周密, 《中学生数 理化》，2017 年第 3 期，32-33。 